(2) Open Access Full Text Article

\title{
Asparagine synthetase expression is associated with the sensitivity to asparaginase in extranodal natural killer/T-cell lymphoma in vivo and in vitro
}

This article was published in the following Dove Press journal:

OncoTargets and Therapy

\author{
Wen-jian Liu' ${ }^{1,2, *}$ \\ Hua Wangl,2,* \\ Xiong-wen Peng ${ }^{1,2, *}$ \\ Wei-da Wang ${ }^{1,2}$ \\ Na-wei Liu ${ }^{1,2}$ \\ Yang Wang ${ }^{1,2}$ \\ Yue $\mathrm{Lu}^{1,2}$ \\ 'Laboratory of Hematology \\ Oncologytate, Key Laboratory \\ of Oncology in South China, \\ Collaborative Innovation Center \\ for Cancer Medicine, Guangzhou, \\ Guangdong 5I0060, People's \\ Republic of China; ${ }^{2}$ Department of \\ Hematologic Oncology, Sun Yat-sen \\ University Cancer Center, Guangzhou, \\ Guangdong 5I0060, People's \\ Republic of China \\ *These authors contributed equally \\ to this work
}

Background: Although asparagine synthetase (AsnS) is associated with drug resistance in leukemia, its function in extranodal natural killer (NK)/T-cell lymphoma (ENKTL) remains unclear.

Methods: The present study investigated the relationship between baseline AsnS mRNA levels and response to asparaginase in ENKTL cell lines. It also determined whether upregulating or downregulating the AsnS mRNA level induces or reverses asparaginase-resistant phenotype.

Results: Interestingly, considerable differences were observed in the sensitivity to asparaginase of the five ENKTL cell lines. The AsnS expression levels were positively correlated with the $\mathrm{IC}_{50}$ values. In addition, the asparaginase resistance was induced or reversed by upregulating or downregulating the AsnS mRNA level in vivo and in vitro. Functional analyses indicated that AsnS did not affect the proliferation and apoptosis of ENKTL cells in the absence of asparaginase.

Conclusion: Together, the data stress the importance of AsnS in the sensitivity to asparaginase in ENKTL and suggest a different therapeutic strategy for patients with a different level of AsnS expression.

Keywords: asparagine synthetase, extranodal natural killer/T-cell lymphomax, asparaginase resistance

\section{Introduction}

The WHO classified extranodal natural killer (NK)/T-cell lymphoma (ENKTL) as a distinct clinicopathologic entity of hematopoietic and lymphoid neoplasms. ${ }^{1,2}$ No optimal treatment strategy has been established for ENKTL, because of its rarity and the limited number of multicenter prospective clinical trials. Anthracycline-based chemotherapeutic regimens have shown inefficacy, probably because of the expression of multidrug-resistant (MDR) genes and their product, P-glycoprotein.

Asparaginase is a bacterial enzyme that hydrolyzes serum asparagine. This enzyme presents a unique antitumor mechanism that is unaffected by P-glycoprotein. The survival of tumor cells that synthesize L-asparagine depends on the plasma level of this amino acid. ${ }^{3}$ The depletion of plasma asparagine inhibits protein synthesis, thereby inhibiting nucleotide synthesis and the subsequent apoptotic cell death of tumor cells. ${ }^{3}$ Asparaginase is an important component of chemotherapeutic regimens for acute lymphoblastic leukemia (ALL)., ${ }^{4,5}$ In addition, asparaginase exhibits antitumor activity against NK cell tumors in vitro and in vivo. ${ }^{6,7}$ In 2000 , Yong et $\mathrm{l}^{8}$ conducted the first effective clinical trial of asparaginase in refractory or relapsed ENKTL. Since then,
Department of Hematologic Oncology, Sun Yat-sen University Cancer Center, 65I Dongfeng Dong Road, Guangzhou, Guangdong 510060, People's Republic of China

Tel +862087342462

Email Ivyuesysucc@163.com 
various asparaginase-based regimens $s^{4,5}$ have emerged as promising treatments for ENKTL. However, a number of patients are resistant to asparaginase, leading to treatment failure and poor prognosis.

The asparagine synthetase (AsnS) gene encodes the enzyme that catalyzes the neosynthesis of asparagine from aspartate, which is an ATP-dependent process that uses glutamine as a nitrogen source. ${ }^{9}$ Several studies have found that asparaginase-resistant cells presented many adaptive changes, including increased AsnS mRNA and protein expression. ${ }^{10,11}$ Aslanian et $\mathrm{al}^{10}$ overexpressed the AsnS protein in MOLT-4 cells by using a Moloney mouse retrovirus system and consequently induced asparaginase resistance in the parental cells. Li et al ${ }^{12}$ found that downregulating the AsnS expression in K562 and Karpas299 cells increased the sensitivity of asparaginase-resistant cells. These studies suggest that the AsnS mRNA level plays a key role in the sensitivity to asparaginase of patients with ALL. However, a few follow-up investigations on patients with ALL patients disputed the general applicability of this hypothesis and the role of the AsnS mRNA level in the sensitivity to asparaginase..$^{13,14}$

Only one study has investigated the AsnS mRNA level in the context of ENKTL, and the results revealed that the AsnS mRNA expression was closely correlated with the sensitivity of lymphoma cell lines to asparaginase in vitro and with the survival of patients with ENKTL. ${ }^{15}$ In the present study, we aim to address two specific questions. First, are baseline AsnS mRNA levels linked to the response to asparaginase in ENKTL cell lines? Second, can the asparaginase-resistant phenotype be induced or reversed by upregulating or downregulating the AsnS mRNA level?

\section{Materials and methods Cell culture}

SNK1, SNK6, SNT8, NKYS, and YTS were EBV-positive cell lines established from primary lesions with nasal NK/ T-cell lymphoma and were provided by Dr Shimizu (Tokyo Medical and Dental University) and was approved by the Institutional Review Board and the ethics committees of Sun Yat-sen University Cancer Center. Also, the human embryonic kidney cell line HEK293T was obtained from the American Type Culture Collection (ATCC) and stored in Sun Yat-sen University Cancer Center. The ENKTL cell lines were cultured in RPMI 1640 medium supplemented with $10 \%$ fetal bovine serum (FBS), 50 units/mL penicillin, $50 \mu \mathrm{g} / \mathrm{mL}$ streptomycin (RPMI 1640 medium, FBS, penicillin, and streptomycin from Gibco, Waltham, MA, USA), and 700 units/mL recombinant human IL-2 (PeproTech, London,
UK) at $37^{\circ} \mathrm{C}$ under $5 \% \mathrm{CO}_{2}$. HEK293T were cultured in DMED medium with $10 \%$ FBS and $1 \%$ Pen-Strep at $37^{\circ} \mathrm{C}$ under $5 \% \mathrm{CO}_{2}$.

\section{Extraction of protein and Western blotting} The cells were lysed in radioimmunoprecipitation assay lysis buffer (Biyuntian, Nanjing, People's Republic of China) containing $1 \%$ phenylmethylsulfonyl. After centrifugation at $14,000 \mathrm{rpm}$ for 10 minutes at $4^{\circ} \mathrm{C}$, the protein content of the supernatant was determined by using the Pierce ${ }^{\mathrm{TM}}$ BCA Protein Assay Kit (Thermo Fisher Scientific, Waltham, MA, USA). The aliquots ( $30 \mu \mathrm{g}$ protein per lane) were separated by $10 \%$ sodium dodecyl sulfate polyacrylamide gel electrophoresis and transferred onto polyvinylidene fluoride membranes (Millipore, Billerica, MA, USA). Then, the membranes containing the protein extracts were probed with a specified primary antibody, followed by either an antirabbit or an antimouse secondary antibody. Signals were detected with enhanced chemiluminescence plus reagents (Millipore). GAPDH was used as the internal control. The primary antibodies consisted of AsnS and GAPDH (Abcam, Cambridge, UK). The secondary HRPconjugated antibodies were antimouse IgG and antirabbit IgG (Cell Signaling Technology, Danvers, MA, USA).

\section{RNA extraction, complementary DNA synthesis, and quantitative real-time polymerase chain reaction}

The total RNAs of the five cells $\left(5 \times 10^{6}\right.$ cells $)$ without asparaginase (Sigma-Aldrich, St Louis, MO, USA) treatment were extracted according to the manufacturer's instructions and reverse-transcribed to complementary DNA (cDNA) by using the PrimeScript ${ }^{\mathrm{TM}}$ RT Reagent Kit (TaKaRa, Kyoto, Japan). Then, quantitative real-time polymerase chain reaction (qRT-PCR) was performed by using the SYBR ${ }^{\circledR}$ Premix Ex Taq ${ }^{\mathrm{TM}}$ II Kit (TaKaRa) on the Roche LightCycler 480 (Roche, Basel, Switzerland). All experiments were conducted in accordance with the manufacturer's protocols. The primer sequences of AsnS were as follows: forward primer 5'-AAAGTGGAGCCTTTTCTTCCTG-3' and reverse primer 5'-AGCCAATCCTTCTGTCTGTCATC-3'. Meanwhile, the primer sequences of human GAPDH were as follows: forward primer 5'-CAGCGACACCCACTCCTC-3' and reverse primer 5'-TGAGGTCCACCACCCTGT-3'. Three parallel analyses were conducted for each sample, and each analysis was repeated three times. The relative expression of the target gene was calculated by inputting the $2^{-\mathrm{ACt}}$ values on the LightCycler 480 software (Roche). 


\section{Cell proliferation assays by CCK-8 and $\mathrm{IC}_{50}$ detection}

The growth rate of the cells was detected by using the Cell Counting Kit-8 (CCK-8) assay (Dojindo, Kumamoto, Japan). Briefly, the cells were collected by centrifugation, washed twice with PBS, and seeded in 96-well plates at a concentration of $1.0 \times 10^{4}$ cells/well. The CCK-8 $(10 \mu \mathrm{L})$ was added into each well at $0,24,48$, and 72 hours of culture. After 2 hours of incubation, the optical density (OD) was detected by a microplate reader at $450 \mathrm{~nm}$, and the proliferation curve was drawn. The actual OD of each well was calculated as follows: $\mathrm{OD}=$ OD value of the test well - average OD value of the blank well.

The CCK-8 was used to detect the drug concentration that inhibits cell growth by $50 \%\left(\mathrm{IC}_{50}\right) ; 1.0 \times 10^{4}$ cells/well were seeded in 96-well plates and then exposed to various doses of asparaginase for 48 hours. Then, $10 \mu \mathrm{L}$ of CCK- 8 reagent was added per well, and the plates were further incubated for 2 hours. The cell survival rate was quantified and plotted against the drug concentration on the basis of the absorbance readings at $450 \mathrm{~nm}$ (A). The OD was assayed to calculate the final cell number. The cell survival rate was calculated as follows: cell viability $(\%)=[\mathrm{A}($ asparaginase $)-\mathrm{A}($ blank $)]$ [A (without asparaginase) - A (blank) $\times 100 \%$. IC $_{50}$ was calculated on Graphpad Prism software.

\section{Cell proliferation and apoptosis assay by flow cytometry}

We also detected the cell proliferation by conducting a 5-ethly-2'-deoxyuridine (EdU) with an iClick ${ }^{\mathrm{TM}}$ EdU Andy Fluor ${ }^{\mathrm{TM}} 647$ flow cytometry Assay Kit (GeneCopoeia, Rockville, MD, USA). The cells were treated with EdU $(10 \mu \mathrm{M})$ for 24 hours and then stained in accordance with the manufacturer's protocols. We detected the cell apoptosis level through flow cytometry by using an Annexin V-PE/7AAD Apoptosis Detection Kit (R\&D Systems, Minneapolis, MN, USA) in accordance with the manufacturer's instructions. This assay enabled the identification of early apoptotic (Annexin V-PE+/7AAD-) cells, late apoptotic/ secondary necrotic (Annexin V-PE+/7AAD+) cells, and primary necrotic (Annexin V-PE-/7AAD+) cells.

\section{Construction of plasmids and recombinant lentiviral vectors}

The plasmids were constructed according to standard methods. All structures were verified with the appropriate restriction digestion and/or sequencing. For the overexpression experiments, human AsnS (NM_003483.4) full-length cDNA was inserted into the lentiviral vector $\mathrm{pHBLV}$-CMVIE-
ZsGreen-T2A-puro by digesting the vector with EcoR I and $\mathrm{Xho} I$ restriction sites. The empty vector served as the negative control. For the knockdown experiments, small hairpin RNAs (shRNAs) directed against the AsnS were inserted into the lentiviral vector pHBLV-U6-ZsGreen-Puro by digesting the vector with BamH I and EcoR I restriction sites.

The AsnS shRNA1 target sequence was 5'-CCGGGCT CTGTTACAATGGTGAAATCTCGAGATTTCACCATTG TAACAGAGCTTTTTG-3', and the AsnS shRNA2 target sequence was 5'-CCGGGTGAACATTATGAAGTCCTTT CTCGAGAAAGGACTTCATAATGTTCACTTTTTG-3' . A scrambled shRNA was used as the control (scramble).

\section{Lentiviral infection and screening of stable cell lines}

High-titer lentiviral vector stocks were packed in HEK293T cells through liposomal-mediated transfections in accordance with the manufacturer's protocol (Clontech Laboratories, Addgene, Cambridge, MA, USA). After 48 hours, the virus was harvested, filtrated, and concentrated by ultrafiltration. The titers of the virus preparations were determined by measuring the number of green fluorescence protein (GFP)-positive HEK293T cells after being infected with several viral solutions accompanied with $8 \mathrm{mg} / \mathrm{mL}$ polybrene (Sigma-Aldrich). The ENKTL cells were infected with the viral solutions at a multiplicity of infection of 90 . For transduction, the cells containing the virus were centrifuged at $1,000 \mathrm{~g}$ for 90 minutes, washed, resuspended in RPMI 1640 medium, and recultivated. The GFP expression was detected by fluorescence microscopy (Nikon, Tokyo, Japan) to determine the infection efficiency. Puromycin ( $2 \mu \mathrm{g} / \mathrm{mL}$; Sigma-Aldrich) was added, and a resistant colony was selected and grown. The overexpression and knockdown efficiency in the transfected cells were confirmed by qRT-PCR and Western blot analysis, as previously described.

\section{Xenograft tumor model and in vivo apoptosis assay by TUNEL}

Six-week-old BALB/c nu/nu mice were maintained in a pathogen-free environment to determine the effect of asparaginase on ENKTL cell growth in a xenograft model. Each group consisted of six mice. The cells $\left(1 \times 10^{7}\right)$ were inoculated subcutaneously into the left flank of the BALB/c $\mathrm{nu} / \mathrm{nu}$ mice. The mice were administered with asparaginase $(2,000 \mathrm{IU} / \mathrm{kg})$ every other day for 2 weeks once the tumor volume was measurable (minor axis $\geq 40 \mathrm{~mm}, \sim 10-14$ days after injection). The changes were observed once every 2 days. The mice were euthanized post experimentation. The tumors were excised and the size was calculated by the 
following formula: volume $=(\pi \times$ length $\times$ width $\times$ height $) / 6$. All animal maintenance and procedures were performed in strict accordance with the recommendations established by the Animal Care and Ethics Committee of Sun Yat-sen University as well as the US National Institutes of Health Guide for the Care and Use of Laboratory Animals. The protocol was approved by the Animal Care and Ethics Committee of Sun Yat-sen University. In animal studies, all efforts were made to minimize the suffering of mice.

Terminal deoxynucleotidyl transferase-mediated dUTPbiotin nick-end labeling (TUNEL) was performed by using the TUNEL Andy Fluor ${ }^{\mathrm{TM}} 594$ Apoptosis Detection Kit (GeneCopoeia) in accordance with the manufacturer's protocol. Briefly, paraffin-embedded tumor samples were cut into $5 \mathrm{~mm}$ sections and mounted on glass slides. After TUNEL, the numbers of apoptotic cells from 15 different fields (five fields from each of the three biological replicates) were determined by fluorescence microscopy (Nikon) to obtain the average number of cells per field.

\section{Statistical analysis}

Statistical analyses were conducted on SPSS 20.0 and GraphPad Prism 6.0 software. All in vitro experiments were performed in triplicate $(n=3)$. Quantitative data were described as mean \pm standard deviation (SD). Two-group comparisons of quantitative data were completed by using the Student's $t$-test. $P$-values of $<0.05$ were considered statistically significant.

\section{Results}

\section{Sensitivity to asparaginase and baseline} AsnS expression in ENKTL cell lines

The CCK-8 assay results revealed that the different lymphoma cell lines displayed significantly varied sensitivities to asparaginase (for 48 hours). Compared with the other cell lines, YTS was considerably more sensitive to asparaginase, with an estimated $\mathrm{IC}_{50}$ of $0.1828 \pm 0.0168 \mathrm{IU} / \mathrm{mL}$. In comparison, $\mathrm{SNK} 1$ had an $\mathrm{IC}_{50}$ of $56.5133 \pm 7.9071 \mathrm{IU} / \mathrm{mL}$. SNK6, SNT8, and NKYS manifested intermediate sensitivities, with approximate $\mathrm{IC}_{50}$ values of $10.3117 \pm 2.2290$, $10.4263 \pm 2.2439$, and $18.1233 \pm 4.2033 \mathrm{IU} / \mathrm{mL}$, respectively (Figure 1A).

We assessed the baseline AsnS mRNA and protein expression in the five ENKTL cell lines. The YTS cells had extremely low levels of AsnS mRNA and protein expression, whereas the SNK1 cells had the highest AsnS mRNA and
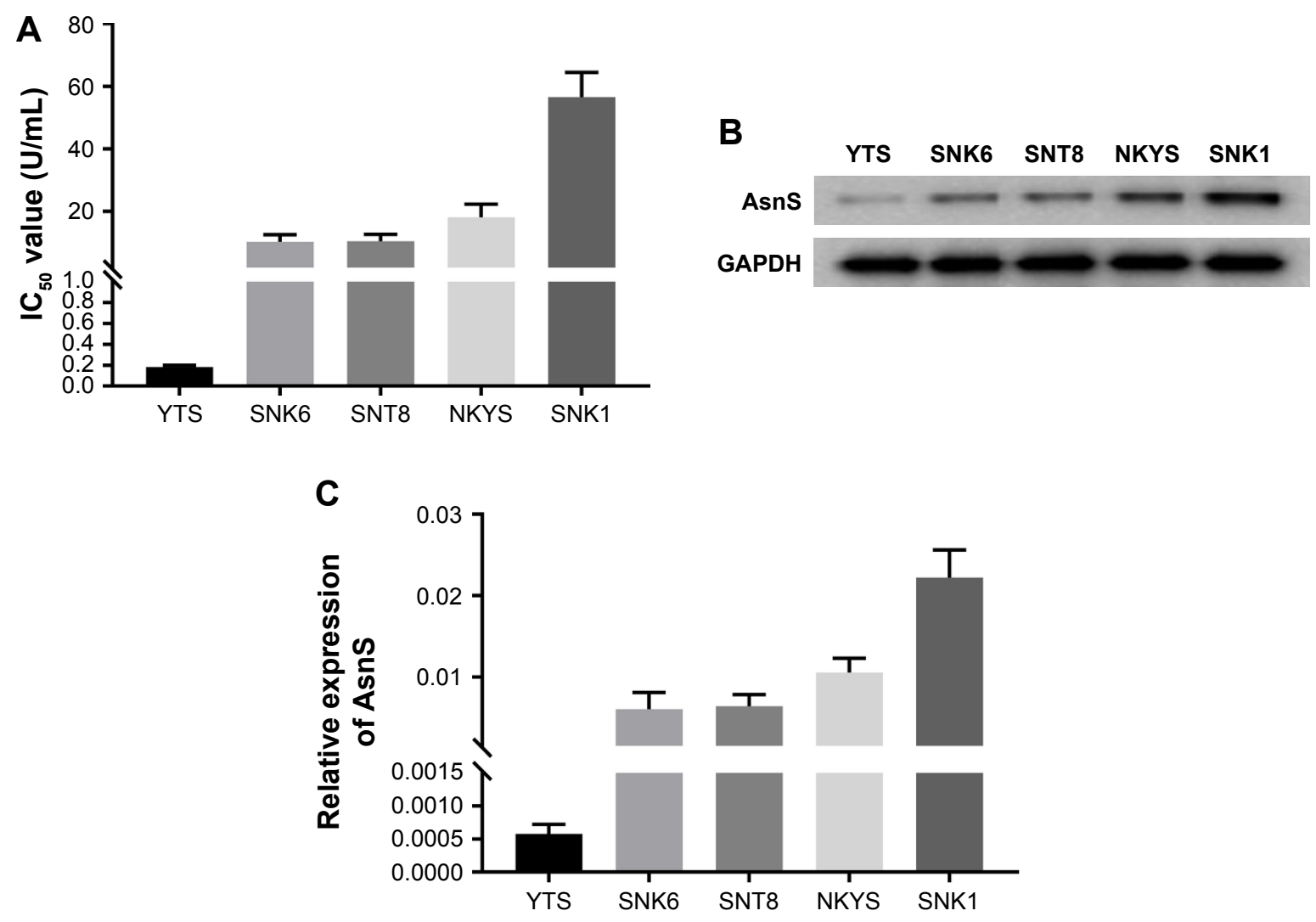

Figure I The AsnS expression levels were positively correlated with the $\mathrm{IC}_{50}$ values in both mRNA and protein.

Notes: $(\mathbf{A}) I_{50}$ values of asparaginase for the 5 ENKTL cell lines. (B) The baseline AsnS protein expression in the five ENKTL cell lines. (C) The baseline AsnS mRNA expression in the five ENKTL cell lines.

Abbreviations: AsnS, asparagine synthetase; ENKTL, extranodal natural killer/T-cell lymphoma. 
protein expression. The AsnS mRNA and protein expression in SNK6, SNT8, and NKYS were intermediate (Figures 1B and $\mathrm{C}$ ). The AsnS expression levels were positively correlated with the $\mathrm{IC}_{50}$ values in both mRNA and protein.

\section{Construction of stable cell lines with AsnS knockdown or overexpression}

Then we determined whether the asparaginase-resistant phenotype was induced or reversed by upregulating or downregulating the AsnS mRNA level. AsnS was overexpressed in the YTS cells (YTS-AsnS-wt, YTS-con as control), which displayed a high sensitivity to asparaginase and a low AsnS mRNA expression level. In contrast, AsnS was downregulated in the SNK1 cells (SNK1-AsnS-sh1, SNK1-AsnS-sh2, SNK1-scramble as control), which exhibited a natural resistance to asparaginase and a high AsnS mRNA expression level. Besides, AsnS was overexpressed and downregulated in the SNT8 cells (SNT8-AsnS-wt, SNT8-con; SNT8-AsnSsh1, SNT8-AsnS-sh2, SNT8-scramble), which showed an intermediate response to asparaginase and an average AsnS mRNA expression level. Then, we screened and concentrated the transfected cells with Puromycin. The stable cell lines were confirmed by fluorescence microscopy, qRT-PCR, and Western blot analysis (Figure 2).

\section{Cell proliferation and apoptosis assay of infected cells without asparaginase}

To identify whether the overexpression or knockdown of AsnS influenced the cell proliferation and apoptosis without asparaginase treatment, a proliferation assay was performed with CCK8 and EdU. Also, an apoptosis assay was conducted by using the Annexin V-PE/7AAD Detection Kit. No significant difference in proliferation and apoptosis was observed between the parental and infected cells without asparaginase (Figure 3), suggesting that the overexpression or knockdown of the AsnS gene did not affect the proliferation and apoptosis of cells without asparaginase treatment.

\section{AsnS is associated with sensitivity to asparaginase in vitro}

The $\mathrm{IC}_{50}$ value of the AsnS overexpression cells (YTS/ AsnS-wt and SNT8/AsnS-wt) was significantly increased, indicating that the sensitivity of AsnS overexpression cells decreased (Figure 4). On the contrary, the $\mathrm{IC}_{50}$ value of the AsnS knockdown cells (SNK1/AsnS-sh1, SNK1/AsnSsh2; SNT8/AsnS-sh1, SNT8/AsnS-sh2) was significantly decreased, indicating that the sensitivity of AsnS knockdown cells increased (Figure 4). These in vitro studies revealed that AsnS was associated with the sensitivity to asparaginase in vitro.

\section{AsnS is associated with sensitivity to asparaginase in vivo}

As for the in vivo experiment, YTS and SNK1 sublines were used to establish the xenografts model, asparaginase was injected as mentioned above once the tumor volume was measurable. As expected, the in vivo xenografts assay also revealed that: AsnS overexpression cells exhibited fast tumor formation, a significant increase in tumor size and weight (Figure 5A) and a decrease in apoptosis rates by TUNEL assay (Figure 5B). AsnS knockdown cells exhibited delayed tumor formation, significant reduction of tumor size and weight (Figure 5C), and an increase in apoptosis rates by TUNEL assay (Figure 5D).

\section{Discussion}

ENKTL is an aggressive type of lymphoma characterized by poor survival, and optimal treatment strategies for this disease have not been established. Anthracycline-based chemotherapy treatments, such as the CHOP regimen, have reportedly yielded unsatisfactory results. ${ }^{16}$ The failure of the CHOP regimen in ENKTL may be attributed to the overexpression of the MDR gene, which increases the P-glycoprotein expression. Doxorubicin and vincristine, which are the main components of the $\mathrm{CHOP}$ regimen, are actively exported by P-glycoprotein, ultimately resulting in inferior treatment outcomes. Chemotherapy regimens based on non-P-glycoprotein efflux medications, such as asparaginase, have been proven as effective alternatives that can overcome P-glycoprotein-mediated MDR and chemotherapy resistance. Asparaginase-based regimens have recently emerged as promising treatments for ENKTL. ${ }^{17-20}$ Unfortunately, a number of patients still experience refractory or relapse, indicating the need to identify the possible asparaginase resistance determinants in ENKTL.

The AsnS mRNA level is considered to play a key role in the sensitivity to asparaginase of patients with ALL. However, no study has verified its role in the context of ENKTL. In the present study, we revealed that the AsnS expression is inversely related to the sensitivity of ENKTL cell lines to asparaginase. Our results were consistent with those of a previous study demonstrating that the AsnS mRNA expression was closely related to the sensitivity of lymphoma cell lines to asparaginase in vitro. ${ }^{15}$ Previous studies revealed that an upregulated AsnS mRNA expression resulted in a simultaneous increase in AsnS protein level and AsnS enzyme activity. ${ }^{10,11}$ Aslanian and Kilberg ${ }^{21}$ confirmed the 
A

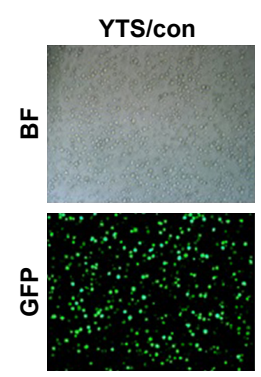

C
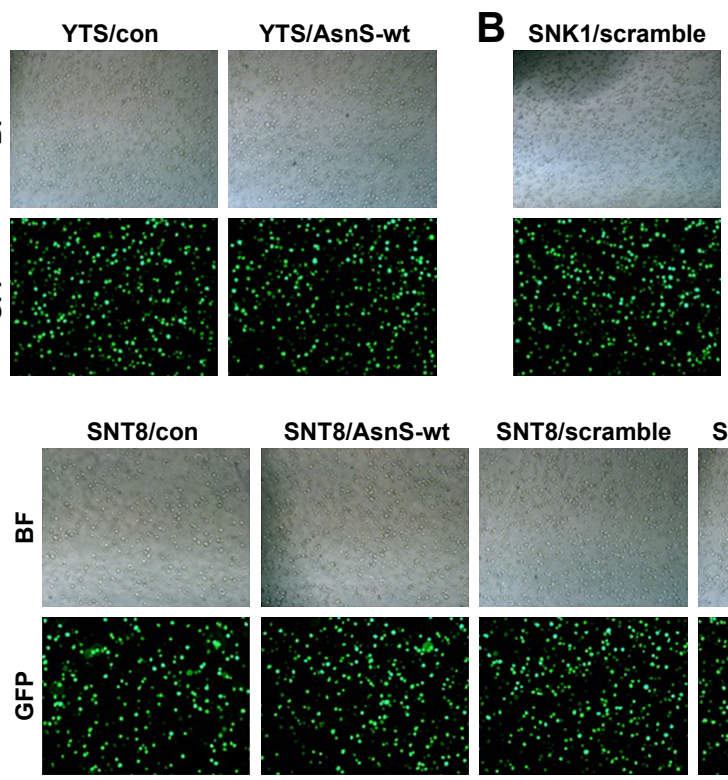

SNK1/AsnS-sh1

SNK1/AsnS-sh2
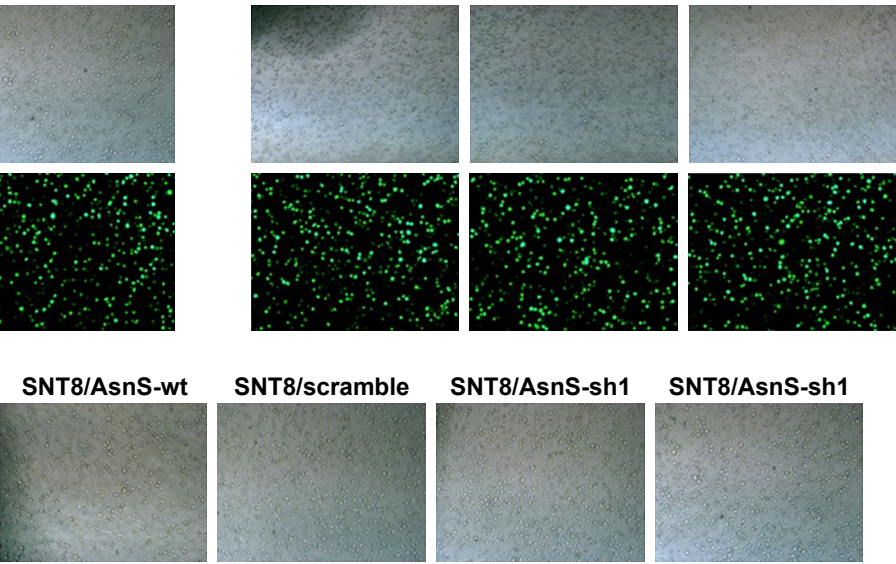

SNT8/AsnS-sh1
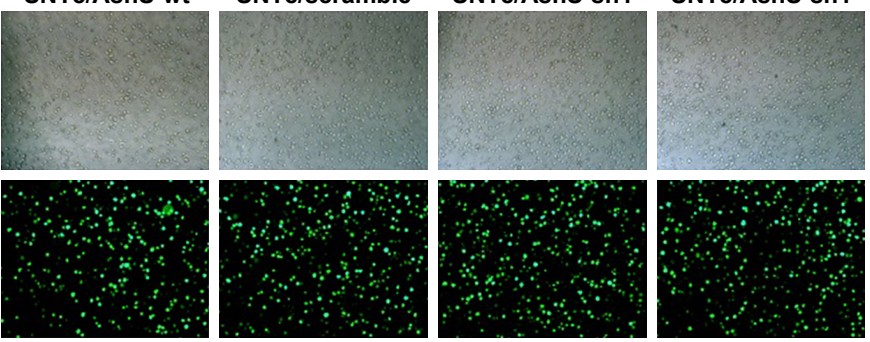

D

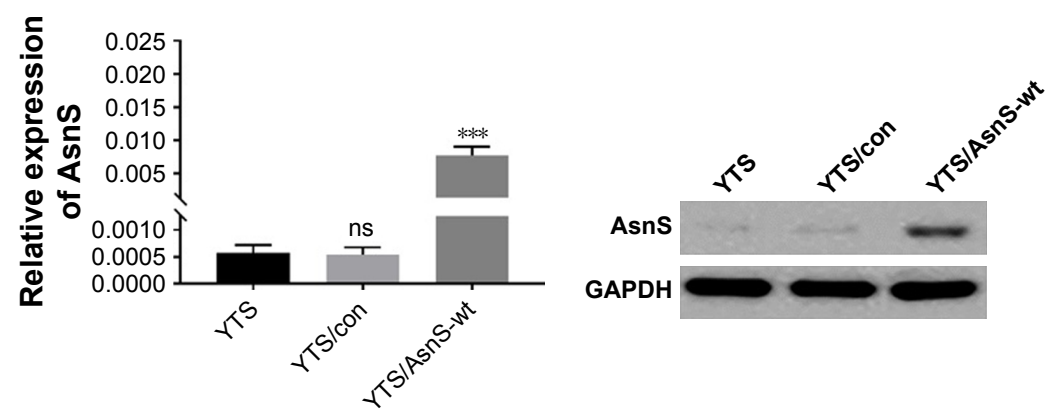

E
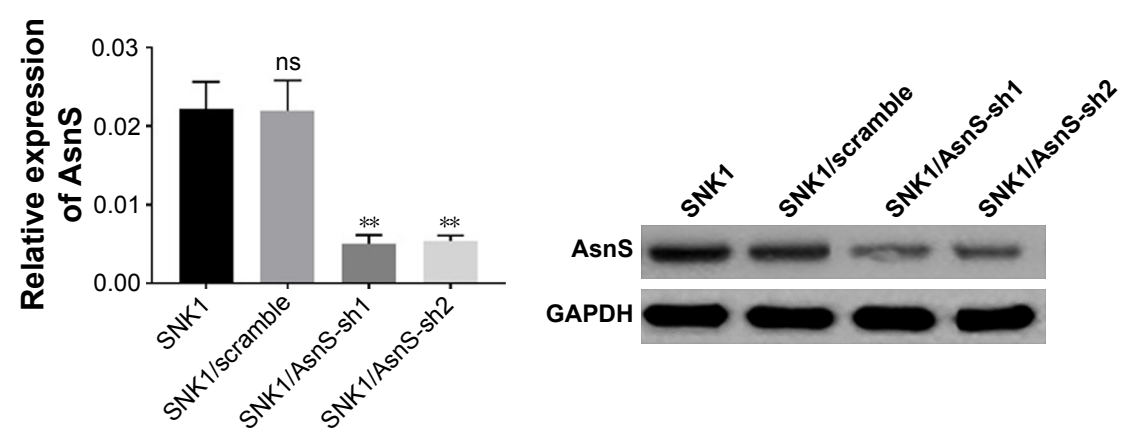

F
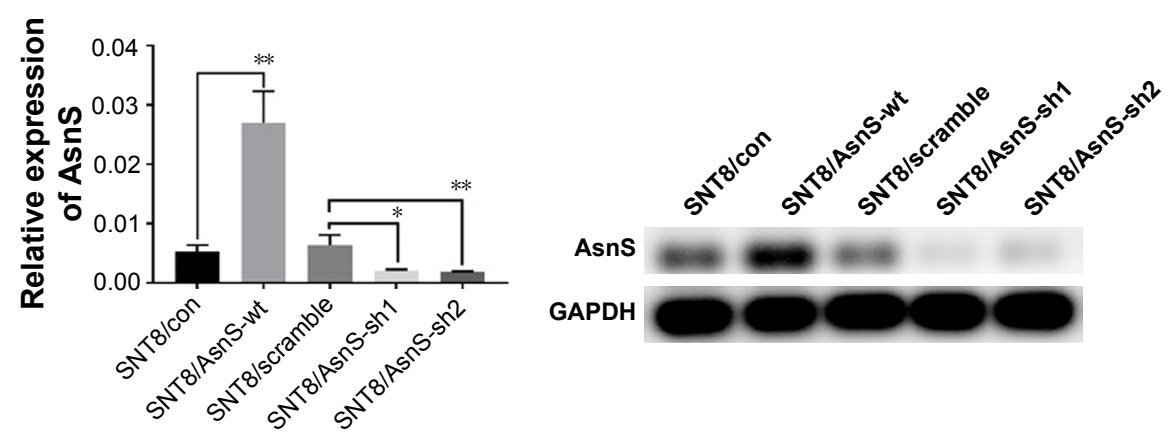

Figure 2 Construction of stable cell lines with AsnS overexpression or knockdown.

Notes: The lentivirus infection efficiency was indicated by BF and GFP fluorescence in (A) YTS/con and YTS/AsnS-wt; (B) SNKI/scramble, SNKI/AsnS-shI, and SNKI/AsnSsh2; (C) SNT8/con, SNT8/AsnS-wt, SNT8/scramble, SNT8/AsnS-shI, and SNT8/AsnS-sh2. qRT-PCR and Western blot analysis of the AsnS overexpression and knockdown efficiency in (D) YTS and its sublines (***P<0.00I vs YTS); (E) SNKI and its sublines $(* * P<0.01$ vs SNKI); (F) SNT8 and its sublines $(* P<0.05$, $* * P<0.0 I$ vs SNT8/con or SNT8/scramble).

Abbreviations: AsnS, asparagine synthetase; BF, bright field; GFP, green fluorescence protein; ns, not significant; qRT-PCR, quantitative real-time polymerase chain reaction. 
direct correlation among the mRNA, protein, and activity levels. Similarly, we found that the AsnS expression levels in protein were positively correlated with those in mRNA.

One reason that the cells expressing low AsnS protein levels were more sensitive to asparaginase treatment was because they produced less asparagine and were, therefore, more dependent on extracellular asparagine to meet metabolic demands. Studies on the putative causes of asparaginase resistance have been performed mostly on ALL cell lines, and the results have been controversial. ${ }^{11,21,22}$
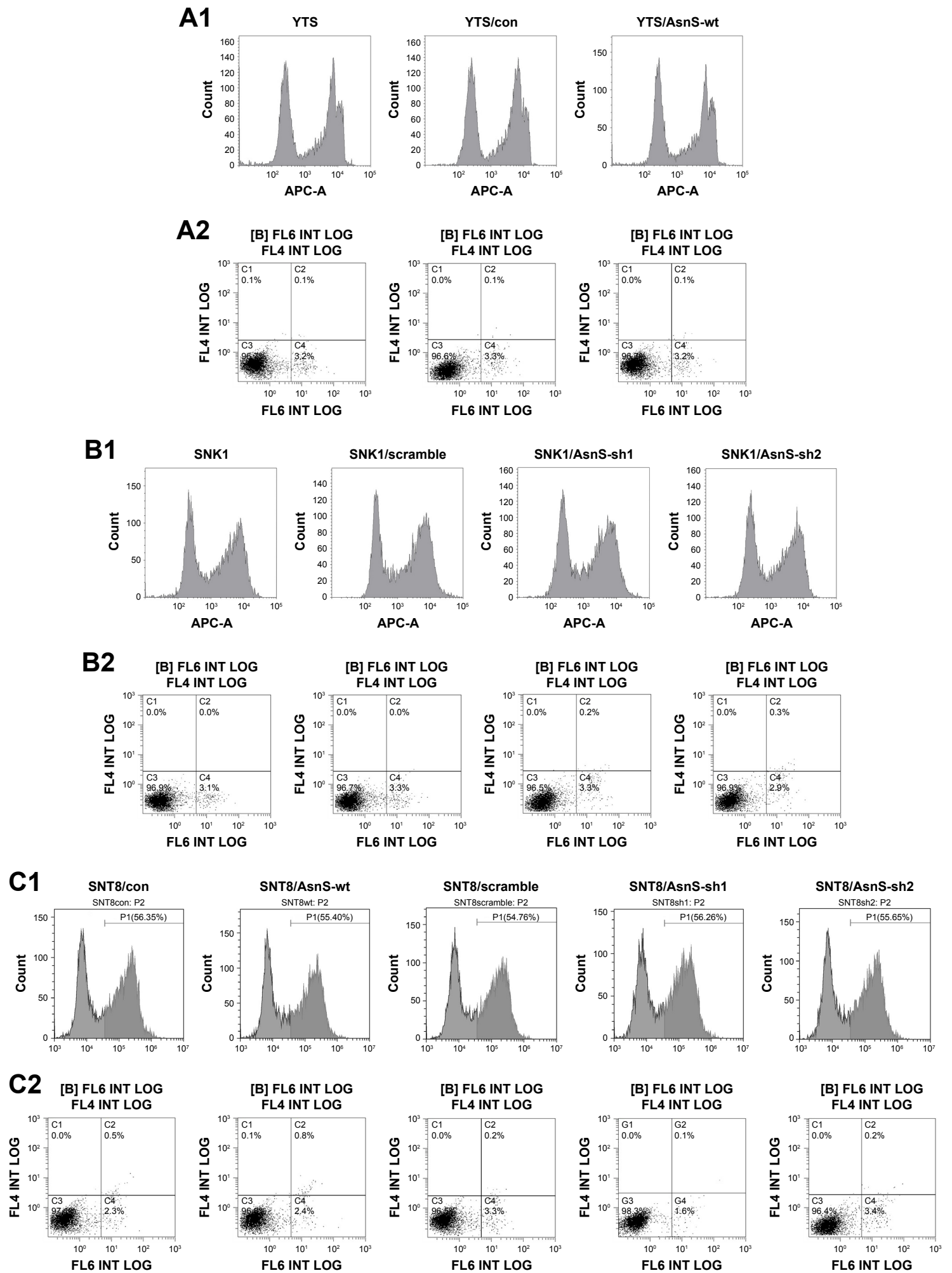

Figure 3 (Continued) 

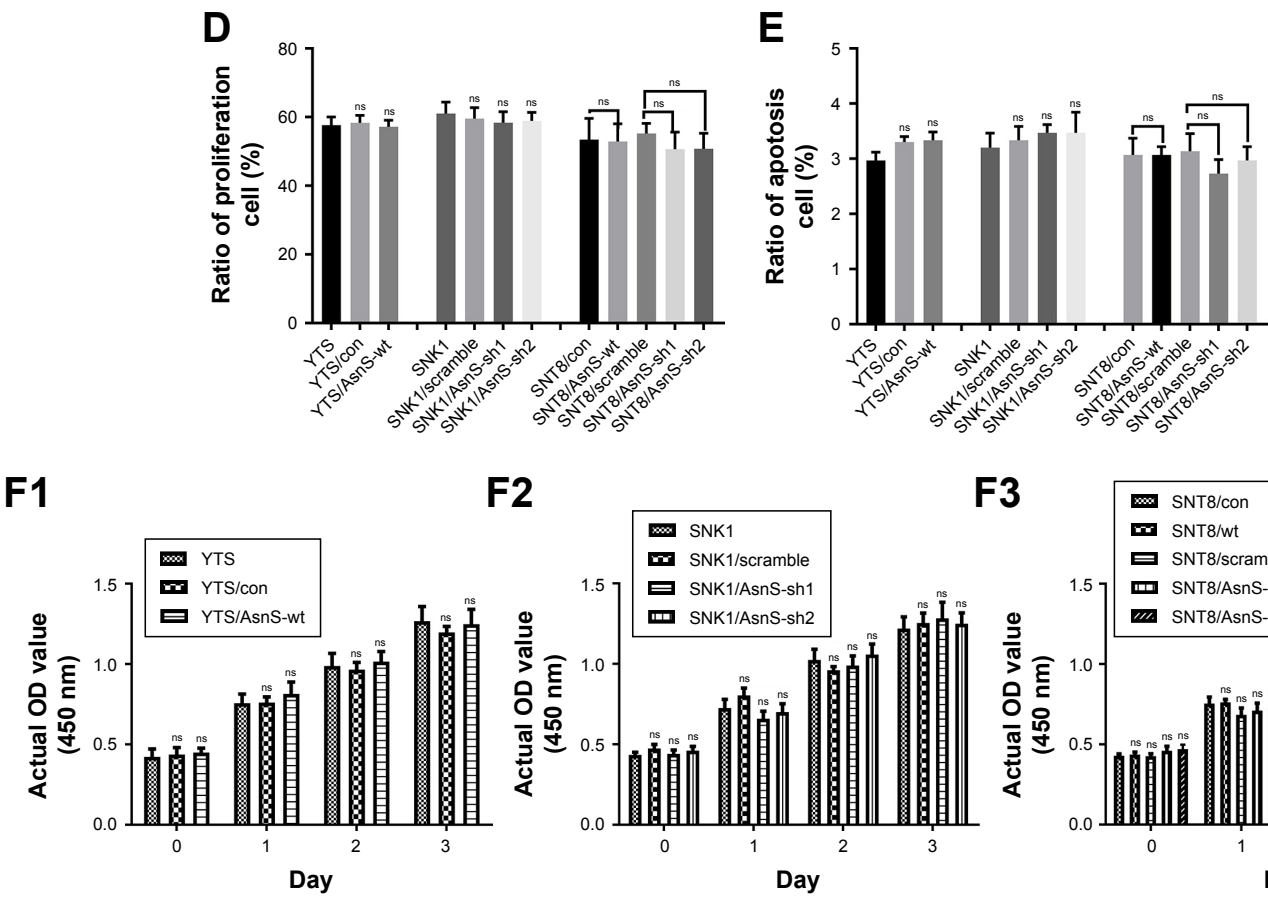

F2

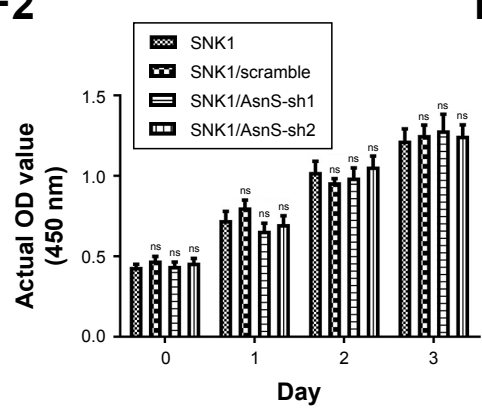

F3

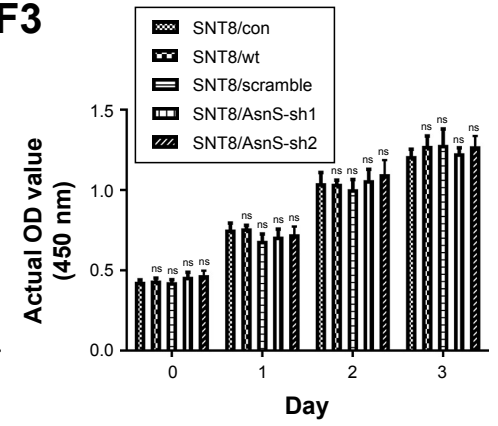

Figure 3 Cell proliferation and apotosis assay of infected cells without asparaginase.

Notes: Proliferation was detected by flow cytometry with the Click-iT EdU Andy Fluor 647 Assay Kit in (AI) YTS, YTS/con, and YTS/AsnS-wt cells; (BI) SNKI, SNKI/ scramble, SNKI/AsnS-shI, and SNKI/AsnS-sh2 cells; (CI) SNT8/con, SNT8/AsnS-wt, SNT8/scramble, SNT8/AsnS-shI, and SNT8/AsnS-sh2. Apotosis was detected by flow cytometry with the annexin V-PE/7AAD Apoptosis Detection Kit in (A2) YTS, YTS/con, and YTS/AsnS-wt cells; (B2) SNKI, SNKI/scramble, SNKI/AsnS-shI, and SNKI/ AsnS-sh2 cells; (C2) SNT8/con, SNT8/AsnS-wt, SNT8/scramble, SNT8/AsnS-shI, and SNT8/AsnS-sh2. (D) No significant difference was observed in proliferation by flow cytometry with the Click-iT EdU Andy Fluor 647 Assay Kit between the parental and infected cells without asparaginase. (E) No significant difference was observed in apoptosis by flow cytometry with the annexin V-PE/7AAD Apoptosis Detection Kit between the parental and infected cells without asparaginase. The proliferation was measured by CCK-8 assay and no significant difference was observed in (FI) YTS, YTS/con, and YTS/AsnS-wt cells; (F2) SNKI, SNKI/scramble, SNKI/AsnS-shI, and SNKI/ AsnS-sh2 cells; (F3) SNT8/con, SNT8/AsnS-wt, SNT8/scramble, SNT8/AsnS-shI, and SNT8/AsnS-sh2. nsp>0.05.

Abbreviations: EdU, 5-ethly-2'-deoxyuridine; AsnS, asparagine synthetase; CCK-8, Cell Counting Kit-8; ns, not significant.

Previous studies have revealed that the AsnS mRNA expression was correlated with asparaginase resistance only in TEL-AML1-negative, but not in TEL-AML1-positive ALL. ${ }^{23}$ In contrast, low AsnS expression in TEL-AML1negative B-lineage is correlated with good prognosis. ${ }^{24}$ Thus, the AsnS level alone did not determine the sensitivity to asparaginase in a number of subtypes of hematological malignancies. Therefore, the role of AsnS in the asparaginase resistance of ALL cells may vary among genetic subtypes. ${ }^{23-25}$ In the present study, we did not examine the genetic aberrations. Further studies may explore whether AsnS expression is related to gene mutations or chromosomal aberrations.

Given that the sensitivity to asparaginase was strongly negatively correlated with the baseline AsnS gene expression in the five ENKTL cell lines, we examined whether the asparaginase-resistant phenotype was induced or reversed by upregulating or downregulating the AsnS mRNA level. SNK1 (highest AsnS mRNA expression level), YTS (lowest AsnS mRNA expression level), and SNT8 (middle AsnS mRNA expression level) were selected for the downregulation and/or upregulation of AsnS mRNA level by lentiviral infection. The AsnS mRNA expression was decreased by using the RNA interference by shRNA. We found that the suppression of AsnS increased the sensitivity to asparaginase in vitro and in vivo. Similarly, the AsnS overexpression decreased the sensitivity to asparaginase in vitro and in vivo. As mentioned, the asparaginase-resistant phenotype was induced or reversed by upregulating or downregulating the AsnS mRNA level in ALL. ${ }^{10,12}$ Moreover, the AsnS knockdown increased the sensitivity to asparaginase in a number of solid tumors, such as hepatocellular carcinoma (HCC) and ovarian cancer. ${ }^{26,27}$ Our results extended the conclusions of previous studies and confirmed the original hypothesis regarding the relationship between the sensitivity to asparaginase and the AsnS expression in ENKTL.

Previous studies have assessed whether AsnS was an oncogene or anti-oncogene. ${ }^{26-29}$ Zhang et $\mathrm{al}^{26}$ demonstrated that AsnS knockdown not only promoted proliferation and tumorigenicity but also enhanced the metastatic potential of $\mathrm{HCC}$, indicating that AsnS was a tumor growth suppressor gene in HCC. In contrast, Patrikainen et $\mathrm{al}^{30}$ and Ameri 
A

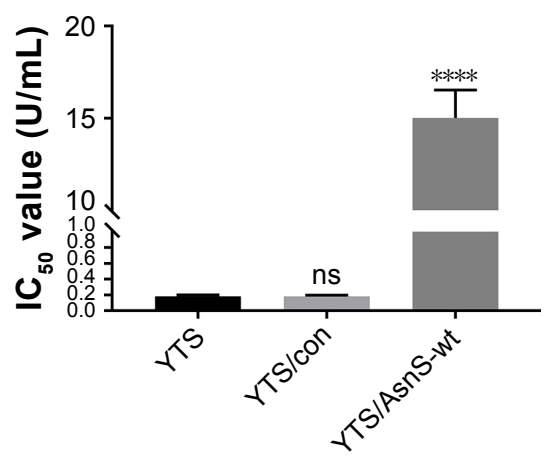

B

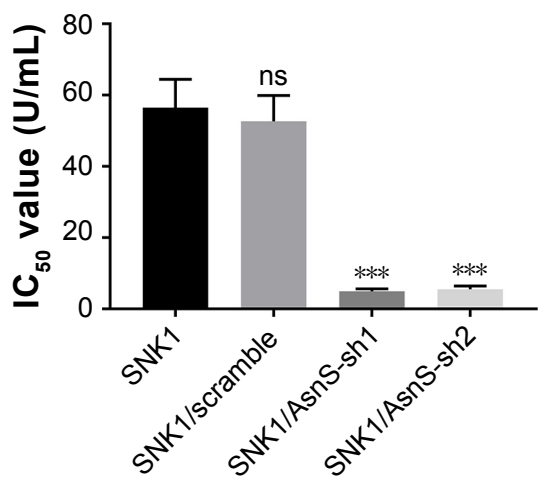

C

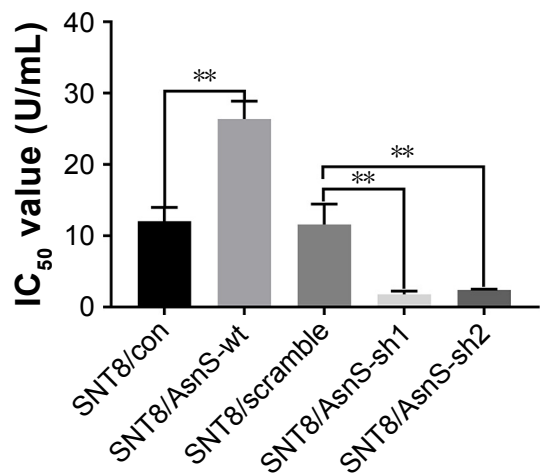

Figure 4 AsnS is associated with the sensitivity to asparaginase in vitro.

Notes: (A) The IC $\mathrm{C}_{50}$ of the YTS, YTS/con, and YTS/AsnS-wt cells. ( ${ }^{n s} P>0.05$, *****P $<0.0001$ vs YTS). (B) The IC ${ }_{50}$ of the SNKI, SNKI/scramble, SNKI/AsnS-shI, and SNKI/ AsnS-sh2 cells. ( ${ }^{\text {ns } P}>0.05$, $* * * P<0.00 I$ vs SNKI). (C) The IC ${ }_{50}$ of the SNT8/con, SNT8/AsnS-wt, SNT8/scramble, SNT8/AsnS-shI, and SNT8/AsnS-sh2 cells (**P<0.0I vs SNT8/con or SNT8/scramble).

Abbreviations: AsnS, asparagine synthetase; ns, not significant.

et $\mathrm{al}^{31}$ revealed that $\mathrm{PC}-3$ prostate cancer cells and human MDA-MB-231 breast cancer cells, which had strong potential for metastasis and increased capacity for colony formation, exhibited elevated AsnS expression, implying that AsnS is a carcinogenic gene in breast and prostate cancers. Our study failed to prove whether AsnS is a tumor growth suppressor or carcinogenic gene in ENKTL, because no significant difference in proliferation and apoptosis was observed between the parental and infected cells in the absence of asparaginase (Figure 3). The conflicting evidence indicates that the roles of AsnS might vary in different tissues or organs, and these differences should be investigated further.

\section{Conclusion}

Our results revealed that the sensitivity to asparaginase is strongly negatively correlated with the baseline AsnS gene expression in ENKTL cell lines. We also demonstrated that asparaginase resistance is induced or reversed
A1

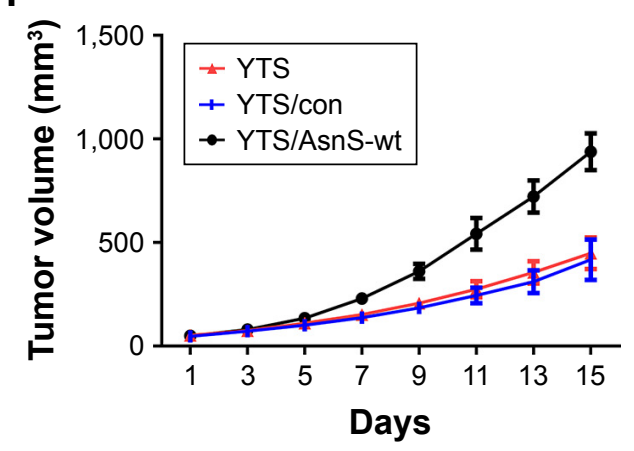

B1

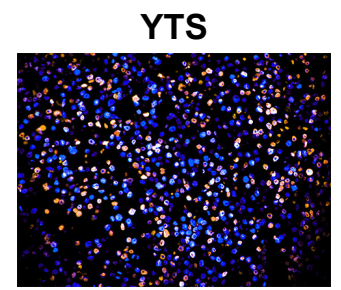

(400x, merge)
A2

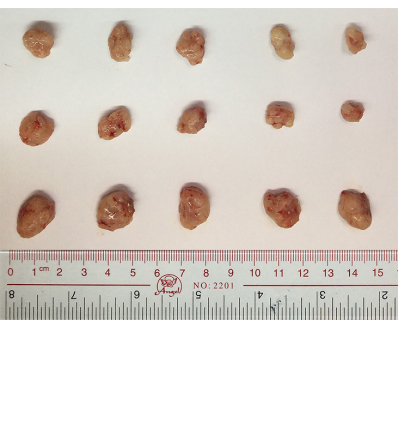

B2

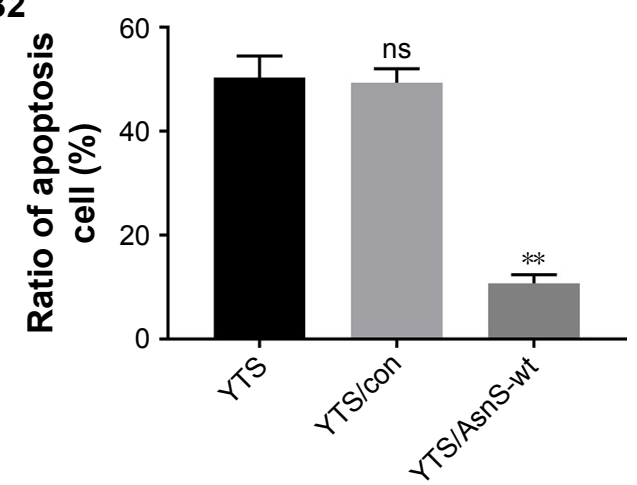

A3

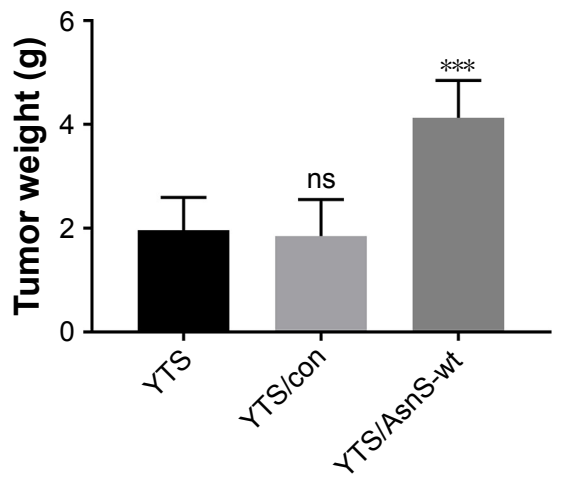

Figure 5 (Continued)

(400x, merge)

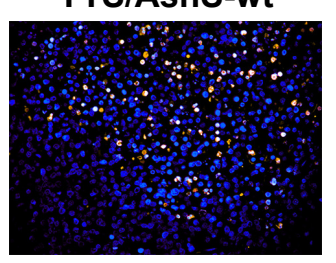

(400x, merge) 

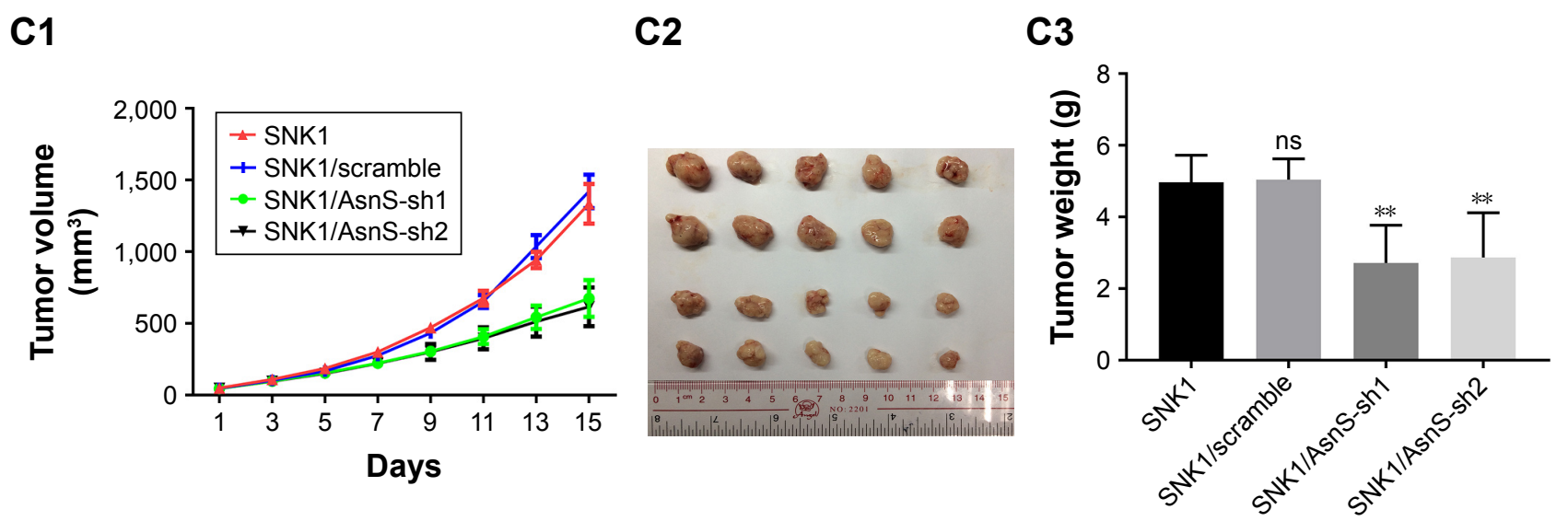

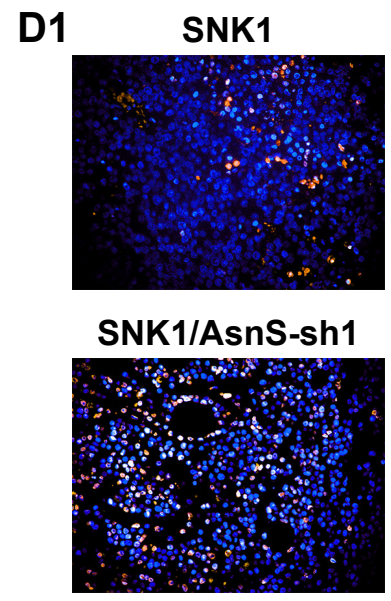

(400x, merge)
SNK1/scramble

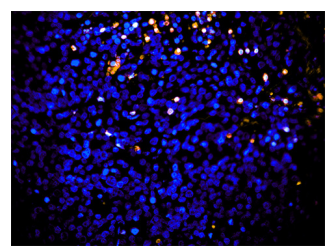

SNK1/AsnS-sh2

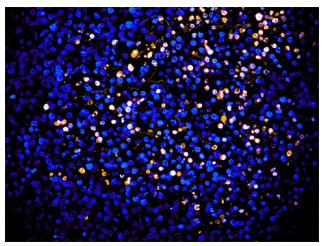

(400x, merge)

D2

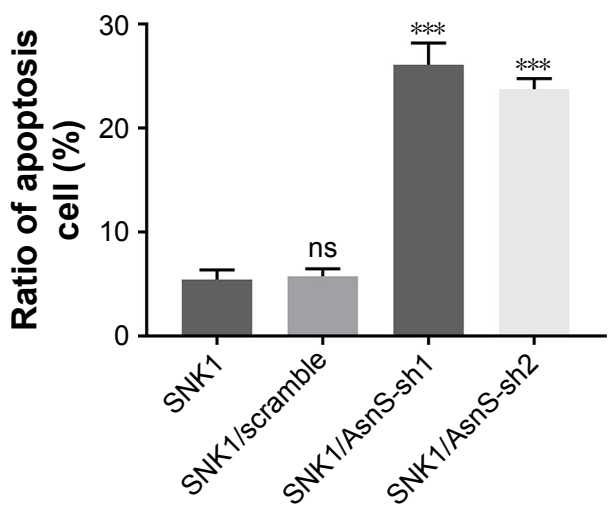

Figure $5 \mathrm{AsnS}$ is associated with the sensitivity to asparaginase in vivo.

Notes: The in vivo xenografts assay revealed that: AsnS overexpression cells exhibited fast tumor formation (AI), a significant increase in tumor size (A2) and weight (A3), and a decrease in apoptosis rates by TUNEL assay (BI and B2) ( ${ }^{\text {ns }} P>0.05, * * P<0.01$, $* * * P<0.00$ I vs $Y$ TS). AsnS knockdown cells exhibited delayed tumor formation ( $\mathbf{C I}$ ), significant reduction of tumor size $(\mathbf{C} 2)$ and weight $(\mathbf{C} 3)$, and an increase in apoptosis rates by TUNEL assay (DI and $\mathbf{D} 2)\left({ }^{n s} P>0.05, * * P<0.01\right.$, $* * * P<0.00 \mathrm{I}$ vs $\left.\mathrm{SNKI}\right)$.

Abbreviations: AsnS, asparagine synthetase; ns, not significant; TUNEL, terminal deoxynucleotidyl transferase-mediated dUTP-biotin nick-end labeling.

by upregulating or downregulating the AsnS mRNA level in vivo and in vitro. Our results suggested that a low AsnS expression should be used in selecting patients for treatment with asparaginase-containing chemotherapy regimens. However, we failed to prove whether AsnS is an oncogene or anti-oncogene in ENKTL. We are also looking forward to the research on the relationship between AsnS expression in ENKTL tissue and treatment response to asparaginasecontaining chemotherapy.

\section{Acknowledgments}

The authors would like to thank the staff members of the Department of Hematologic Oncology, Cancer Center, Sun Yat-sen University, for their valuable suggestions and assistance. This work was supported by the National Natural Science Foundation of China under Grant (Number 81272620) and Guangdong Provincial Science and Technology Projects under Grant (Number 2016A020215086).

\section{Disclosure}

The authors report no conflicts of interest in this work.

\section{References}

1. Tohda S. Overview of lymphoid neoplasms in the fourth edition of the WHO classification. Rinsho Byori. 2012;60(6):560-564.

2. Swerdlow SH, Campo E, Pileri SA, et al. The 2016 revision of the World Health Organization classification of lymphoid neoplasms. Blood. 2016;127(20):2375-2390.

3. Cooney DA, Handschumacher RE. L-asparaginase and L-asparagine metabolism. Anпu Rev Pharmacol. 1970;10:421-440.

4. Liu WJ, Wang H, Wang WD, et al. Use of PEG-asparaginase in newly diagnosed adults with standard-risk acute lymphoblastic leukemia compared with E. coli-asparaginase: a retrospective single-center study. Sci Rep. 2016;6:39463.

5. Avramis VI. Asparaginases: a successful class of drugs against leukemias and lymphomas. J Pediatr Hematol Oncol. 2011;33(8):573-579.

6. Obama K, Tara M, Niina K. L-asparaginase-Based induction therapy for advanced extranodal NK/T-cell lymphoma. Int J Hematol. 2003; 78(3):248-250.

7. Ando M, Sugimoto K, Kitoh T, et al. Selective apoptosis of natural killer-cell tumours by 1-asparaginase. Br J Haematol. 2005;130(6): 860-868. 
8. Yong W, Zhang Y, Zheng W. The efficacy of L-asparaginase in the treatment of refractory midline peripheral T-cell lymphoma. Zhonghua Xиe Ye Xue Za Zhi. 2000;21(11):577-579.

9. Richards NG, Schuster SM. Mechanistic issues in asparagine synthetase catalysis. Adv Enzymol Relat Areas Mol Biol. 1998;72:145-198.

10. Aslanian AM, Fletcher BS, Kilberg MS. Asparagine synthetase expression alone is sufficient to induce 1-asparaginase resistance in MOLT-4 human leukaemia cells. Biochem J. 2001;357(Pt 1):321-328.

11. Hutson RG, Kitoh T, Moraga Amador DA, Cosic S, Schuster SM, Kilberg MS. Amino acid control of asparagine synthetase: relation to asparaginase resistance in human leukemia cells. Am J Physiol. 1997; 272(5 Pt 1):C1691-C1699.

12. Li BS, Gu LJ, Luo CY, et al. The downregulation of asparagine synthetase expression can increase the sensitivity of cells resistant to 1-asparaginase. Leukemia. 2006;20(12):2199-2201.

13. Hermanova I, Zaliova M, Trka J, Starkova J. Low expression of asparagine synthetase in lymphoid blasts precludes its role in sensitivity to L-asparaginase. Exp Hematol. 2012;40(8):657-665.

14. Appel IM, den Boer ML, Meijerink JP, Veerman AJ, Reniers NC, Pieters R. Up-regulation of asparagine synthetase expression is not linked to the clinical response L-asparaginase in pediatric acute lymphoblastic leukemia. Blood. 2006;107(11):4244-4249.

15. Li Y, Zhang X, Hu T, et al. Asparagine synthetase expression and its potential prognostic value in patients with NK/T cell lymphoma. Oncol Rep. 2014;32(2):853-859.

16. Kim WS, Song SY, Ahn YC, et al. CHOP followed by involved field radiation: is it optimal for localized nasal natural killer/T-cell lymphoma? Ann Oncol. 2001;12(3):349-352.

17. Yamaguchi M, Kwong YL, Kim WS, et al. Phase II study of SMILE chemotherapy for newly diagnosed stage IV, relapsed, or refractory extranodal natural killer (NK)/T-cell lymphoma, nasal type: the NK-Cell Tumor Study Group study. J Clin Oncol. 2011;29(33):4410-4416.

18. Kwong YL, Kim WS, Lim ST, et al. SMILE for natural killer/T-cell lymphoma: analysis of safety and efficacy from the Asia Lymphoma Study Group. Blood. 2012;120(15):2973-2980.

19. Jaccard A, Gachard N, Marin B, et al; GELA and GOELAMS Intergroup. Efficacy of L-asparaginase with methotrexate and dexamethasone (AspaMetDex regimen) in patients with refractory or relapsing extranodal NK/T-cell lymphoma, a phase 2 study. Blood. 2011;117(6): $1834-1839$.
20. Wang L, Wang ZH, Chen XQ, et al. First-line combination of gemcitabine, oxaliplatin, and L-asparaginase (GELOX) followed by involvedfield radiation therapy for patients with stage IE/IIE extranodal natural killer/T-cell lymphoma. Cancer. 2013;119(2):348-355.

21. Aslanian AM, Kilberg MS. Multiple adaptive mechanisms affect asparagine synthetase substrate availability in asparaginase-resistant MOLT-4 human leukaemia cells. Biochem J. 2001;358(Pt 1):59-67.

22. Fine BM, Kaspers GJ, Ho M, Loonen AH, Boxer LM. A genome-wide view of the in vitro response to 1-asparaginase in acute lymphoblastic leukemia. Cancer Res. 2005;65(1):291-299.

23. Stams WA, den Boer ML, Beverloo HB, et al. Sensitivity to L-asparaginase is not associated with expression levels of asparagine synthetase in $\mathrm{t}(12 ; 21)+$ pediatric ALL. Blood. 2003;101(7):2743-2747.

24. Stams WA, den Boer ML, Holleman A, et al. Asparagine synthetase expression is linked with L-asparaginase resistance in TEL-AML1negative but not TEL-AML1-positive pediatric acute lymphoblastic leukemia. Blood. 2005;105(11):4223-4225.

25. den Boer ML, Evans WE, Pieters R. TELAML1-positive ALL: a discordant genotype. Cell Cycle. 2005;4(8):997-998.

26. Zhang B, Dong LW, Tan YX, et al. Asparagine synthetase is an independent predictor of surgical survival and a potential therapeutic target in hepatocellular carcinoma. Br J Cancer. 2013;109(1):14-23.

27. Lorenzi PL, Reinhold WC, Rudelius M, et al. Asparagine synthetase as a causal, predictive biomarker for L-asparaginase activity in ovarian cancer cells. Mol Cancer Ther. 2006;5(11):2613-2623.

28. Cui H, Darmanin S, Natsuisaka M, et al. Enhanced expression of asparagine synthetase under glucose-deprived conditions protects pancreatic cancer cells from apoptosis induced by glucose deprivation and cisplatin. Cancer Res. 2007;67(7):3345-3355.

29. Sircar K, Huang H, Hu L, et al. Integrative molecular profiling reveals asparagine synthetase is a target in castration-resistant prostate cancer. Am J Pathol. 2012;180(3):895-903.

30. Patrikainen L, Porvari K, Kurkela R, Hirvikoski P, Soini Y, Vihko P. Expression profiling of PC-3 cell line variants and comparison of MIC-1 transcript levels in benign and malignant prostate. Eur J Clin Invest. 2007;37(2):126-133.

31. Ameri K, Luong R, Zhang H, et al. Circulating tumour cells demonstrate an altered response to hypoxia and an aggressive phenotype. Br J Cancer. 2010;102(3):561-569.
OncoTargets and Therapy

\section{Publish your work in this journal}

OncoTargets and Therapy is an international, peer-reviewed, open access journal focusing on the pathological basis of all cancers, potential targets for therapy and treatment protocols employed to improve the management of cancer patients. The journal also focuses on the impact of management programs and new therapeutic agents and protocols on

\section{Dovepress}

patient perspectives such as quality of life, adherence and satisfaction. The manuscript management system is completely online and includes a very quick and fair peer-review system, which is all easy to use. Visit http://www.dovepress.com/testimonials.php to read real quotes from published authors. 